\title{
Cachet Dosing Unit
}

National Cancer Institute

\section{Source}

National Cancer Institute. Cachet Dosing Unit. NCI Thesaurus. Code C149364.

A unit of presentation used to represent the quantity of product that is found in a single discrete entity where the pharmaceutical dose form is a type of cachet. 